Results A total of 767 (92.9\%) men were included in the study. The mean age was 26.5 (SD 8.3) years old. Prevalence of Chlamydia infection was $13.1 \%$ (95\% CI $10.7 \%$ to $15.5 \%$ ) and gonorrhoea was $18.4 \%$ (95\% CI $15.7 \%$ to $21.1 \%$ ). Coinfection prevalence was $4.4 \%$ ( $95 \%$ CI $2.95 \%$ to $5.85 \%$ ) in men who sought attendance in STI clinics. Factors identified as associated with $C$ trachomatis were younger age $(15-24)(\mathrm{OR}=1.4(95 \% \mathrm{CI} 1.01$ to 1.91$))$, present urethral discharge $(\mathrm{OR}=4.8$ (95\% CI 1.52 to 15.05$)$ ), genital warts (OR=3.0 (95\% CI 1.49 to 5.92$)$ ) and previous history of urethral discharge ( $\mathrm{OR}=2.4$ (95\% CI 1.11-5.18)]. Variables associated with gonorrhoea were younger age (15 to 24$)[\mathrm{OR}=1.5$ (95\% CI 1.09-2.05)], presence of urethral discharge $[\mathrm{OR}=9.9$ (95\% CI 5.53-17.79)], genital warts $[\mathrm{OR}=18.3$ (95\% CI 8.03$41.60)]$ and ulcer present upon clinical examination $[\mathrm{OR}=4.9(95 \% \mathrm{CI}$ 1.06-22.73)]. Conclusions-These findings have important implications for education and prevention actions directed towards men at risk of HIV/STD. A venue-based approach to offer routine screening for young men in STD clinics should be stimulated.

\section{P1-S1.09 TRENDS IN THE AETIOLOGY OF SEXUALLY TRANSMITTED INFECTIONS AND HIV COINFECTIONS AMONG STI PATIENTS ATTENDING ALEXANDRA HEALTH CENTRE, JOHANNESBURG, SOUTH AFRICA (2007-2010)}

doi:10.1136/sextrans-2011-050108.9

D Lewis, C Ricketts, N Bhojraj, G de Gita, P Magooa, I Venter, L Mshibe, A Vezi, E Muller, F Radebe. NICD/NHLS, Sandringham, South Africa

Objectives To determine trends in the relative prevalence of aetiologies of urethral discharge (UDS), vaginal discharge (VDS) and genital ulcer (GUS) syndromes, and in the seroprevalence of syphilis, HSV-2 and HIV.

Methods Consecutive male (UDS/GUS) and female (VDS/GUS) patients were enrolled at Alexandra Health Centre, Johannesburg from January to April each year during 2007-2010. Urethral swabs (UDS), endocervical swabs/vaginal smears (VDS), genital ulcer swabs/smears (GUS) and sera (all) were collected with written informed consent. Real-time PCR assays were used to detect Neisseria gonorrhoeae (NG), Chlamydia trachomatis (CT), Trichomonas vaginalis (TV) and Mycoplasma genitalium (MG) from UDS/VDS swabs, and herpes simplex virus (HSV), Treponema pallidum (TP) Haemophilus ducreyi (HD) and Chlamydia trachomatis L1-3 (LGV) from ulcer swabs. Slides were stained for bacterial vaginosis/candidiasis (BV/CA, VDS) and granuloma inguinale (GI, GUS). Sera were tested for syphilis (rapid plasmin reagin, RPR; Omega Diagnostics), for HSV-2 (HerpeSelect IgG; Focus Diagnostics) and for HIV (Determine; Abbott Laboratories). $\chi^{2}$ for linear trend analyses were undertaken with summary data (Prism v.2, GraphPad Software).

Results 928 UDS, 805 VDS and 455 GUS patients were recruited overall. Trends in the relative prevalence of most syndrome aetiologies were non-significant between 2007 and 2011-NG (UDS, 71\%-79\%; VDS 11\%-17\%), CT (UDS, 20\%-25\%; VDS, 27\%-37\%), MG (UDS, 10\%-13\%; VDS, 11\%-14\%), BV (VDS, 30\%-36\%), CA (VDS, $26 \%-$ $31 \%$ ), HSV (GUS, $53 \%-75 \%$ ), TP (GUS, $4 \%-7 \%$ ), HD (GUS, $0 \%-$ $2 \%)$, LGV $(0 \%-2 \%)$. There were no cases of GI. There was, however, significant decreasing trends for TV detection among UDS $(4 \%-13 \%$, $p=0.003)$ and VDS $(19 \%-34 \%, p=0.001)$ patients. Serologically, VDS patients had a decreasing trend in RPR seropositivity (1-8\%, $\mathrm{p}<0.001)$ and, importantly, HIV coinfections decreased among both UDS $(29 \%-39 \%, \mathrm{p}=0.011)$ and GUS $(60 \%-75 \%, \mathrm{p}=0.032)$ patients. Non-significant variations in seropositivity were observed for RPR tests among UDS $(1 \%-3 \%)$ and GUS $(4 \%-11 \%)$ patients, for HSV-2 among all groups (UDS, 50\%-60\%; VDS, 74\%-84\%; GUS, $81 \%-$ $87 \%$ ), and for HIV among VDS (48\%-59\%) patients.

Conclusions These data suggest significant decreases in the prevalence of HIV coinfection in UDS/GUS patients and of trichomoniasis as a cause of UDS/VDS. Though the HIV trends are encouraging for men, the lack of a similar trend for women with VDS is of public health concern.

\section{P1-S1.10 MICROBIOLOGICAL SURVEILLANCE FOR SEXUALLY TRANSMITTED INFECTIONS IN WINDHOEK AND OSHAKATI, NAMIBIA (2007)}

doi:10.1136/sextrans-2011-050108.10

${ }^{1} \mathrm{~S}$ Tobias, ${ }^{2} \mathrm{~S}$ Shonhiwa, ${ }^{1} \mathrm{~F}$ Norbert, ${ }^{3} \mathrm{O}$ Oke, ${ }^{4} \mathrm{C}$ Ndjavera, ${ }^{4} \mathrm{~S}$ Chikukwa, ${ }^{5} \mathrm{~F}$ Radebe, ${ }^{5}$ D Lewis. ${ }^{1}$ Ministry of Health \& Social Services, Windhoek, Namibia; ${ }^{2}$-Tech Namibia, Namibia; ${ }^{3}$ WHO, Namibia; ${ }^{4}$ Namibian Institute of Pathology, Namibia; ${ }^{5}$ NICD/NHLS, South Africa

Background A microbiological survey was undertaken to enable review of Namibia's syndromic management-based STI treatment guidelines (1999).

Methods This Government-approved survey took place at Katutura Health Centre (Windhoek) and Oshakati Intermediate Hospital over 9 months in 2007. Consecutive patients with urethral discharge (UDS), vaginal discharge (VDS) and genital ulcer (GUS) syndromes gave verbal consent to provide anonymous samples for the following tests-(i) real-time PCR (RT-PCR) for Neisseria gonorrhoeae (NG), Chlamydia trachomatis (CT), Trichomonas vaginalis (TV), Mycoplasma genitalium (MG) (UDS, urethral swabs; VDS, endocervical swabs) (ii) RT-PCR detection of herpes simplex virus (HSV), Treponema pallidum (TP), Haemophilus ducreyi (HD), Chlamydia trachomatis L1-L3 (LGV) (ulcer swabs), (iii) Giemsa staining of ulcer smears for granuloma inguinale (GI), (iv) Gram staining of vaginal smears for Candida (CA) and bacterial vaginosis (BV), (v) NG culture and determination of ciprofloxacin and ceftriaxone minimum inhibitory concentrations (MIC) by E test (urethral swabs), and (vi) HIV ELISA (blood). All patients were provided with STI syndromic treatment, offered same-day HIV counselling and testing, and partner notification was discussed. Data analysis was performed in STATA v10 and the $\chi^{2}$ test used to assess difference by survey site.

Results 199 UDS, 200 VDS and 199 GUS episodes were surveyed among 598 patients (293, Windhoek; 305, Oshakati). The relative prevalence of pathogens/conditions by syndrome was-(i) UDS-NG 87\% (173), CT 9\% (18), TV 5\% (9), MG 2\% (4), (ii) VDS-NG 5\% (9), CT 8\% (15), TV 15\% (29), MG 4\% (8), BV 65\% (127), CA 17\% (33), and (iii) GUS-HSV 49\% (97), TP 3\% (5), LGV $2 \%(2)$, GI 0.5\% (1), HD 0\% (0). Syndrome-specific HIV prevalence was 36\% (62) for UDS, 29\% (57) for VDS and 53\% (91) for GUS. Ciprofloxacin resistance (MIC=1) was detected in $28(24 \%)$ of 118 viable NG isolates; all isolates were deemed susceptible to ceftriaxone. Ciprofloxacin resistance was significantly higher in Oshakati compared to Windhoek (48\% vs 5\%, p<0.001).

Conclusions The survey highlighted the importance of gonorrhoea, genital herpes, HIV-coinfection and, in Oshakati, the high prevalence of ciprofloxacin resistant NG. As a result of this survey, Namibia's STI guidelines were revised in 2008 by adding acyclovir to existing GUS treatment and replacing ciprofloxacin with oral cefixime as treatment for presumptive gonorrhoea.

\section{P1-S1.11 PREVALENCE OF TRICHOMONAS VAGINALIS, CHLAMYDIA AND GONORRHOEA IN WOMEN AT THE MIAMI-DADE COUNTY HEALTH DEPARTMENT STD CLINIC}

doi:10.1136/sextrans-2011-050108.11

${ }^{1} \mathrm{H}$ Tookes, ${ }^{1} \mathrm{~N}$ Hirsch, ${ }^{1} \mathrm{C}$ Diaz, ${ }^{2} \mathrm{O}$ Ponomareva, ${ }^{1} \mathrm{~J} \mathrm{G}$ Castro. ${ }^{1}$ University of Miami Miller School of Medicine, Miami, USA; ${ }^{2}$ Miami Dade County Health Department, Miami, USA

Background Miami, Florida, has the highest rate of HIV diagnoses in the USA (70.3 per 100000 people), accounting for 1218 of the 41269 
incident HIV cases nationwide (2008). Although Trichomonas vaginalis (TV) infection has been associated with an increased risk of HIV acquisition in women, the prevalence of TV in Miami is unknown due to lack of routine screening and low sensitivity testing methods. The purpose of this study is to establish a prevalence of TV in women seeking services at the Miami-Dade County Health Department Downtown STD Clinic compared to the prevalence of routinely screened STDs, gonorrhoea (GC) and Chlamydia (CT).

Methods This study analyses baseline data from the Miami site of Project AWARE which is a multi-centre randomised clinical trial that seeks to test the effectiveness of risk reduction counselling in preventing sexually transmitted infections including HIV among HIV-negative persons. Eligibility criteria for Project Aware included negative or unknown HIV status, age of 18 , and ability to provide informed consent. We recruited 251 women aged 18-64 for STD screening. The screen included FDA-approved Aptima GC/CT NAAT (nucleic acid amplification test) and a newly validated Aptima TV NAAT. Specimens were collected by vaginal swab performed either by a clinician or the patient. Additionally, we report the TV diagnoses given the current clinic protocol in which only symptomatic women received a wet mount analysed by light microscopy. We use descriptive statistics to report the prevalence of GC, CT and TV (as diagnosed by NAAT and wet mount) in our clinic.

Results Of the 251 women in our study, 163 high-risk populations. Received a wet mount. By wet mount, we found a $9 \%$ prevalence of TV in our patients. Comparatively, when all 251 patients were screened using NAAT, the TV prevalence was $20 \%$. The prevalence of CT was $14 \%$ and GC was $6 \%$.

Conclusions TV is the most prevalent STD in patients in our clinic which draws upon high-risk individuals in urban Miami. TV infections were greater than CT, often believed to be the most prevalent STD in our population. Further, the routine screening using the TV NAAT detected 27 cases of TV that would have been undiagnosed given current clinic protocol. Since Miami, FL has the highest prevalence of HIV in the country and TV infection has been linked to new HIV infection, we believe that routine TV screening by NAAT should be instituted in high-risk populations.

\section{P1-S1.12 AGE-SPECIFIC INCIDENCE OF CHLAMYDIA, GONORRHOEA, AND TRICHOMONIASIS INFECTION IN ADOLESCENT WOMEN}

doi:10.1136/sextrans-2011-050108.12

${ }^{1} \mathrm{~W}$ Tu, ${ }^{1} \mathrm{~B}$ Batteiger, ${ }^{1} \mathrm{~S}$ Ofner, ${ }^{2} \mathrm{~B}$ Van Der Pol, ${ }^{1} \mathrm{D}$ Fortenberry. ${ }^{1}$ Indiana University, School of Medicine, Indianapolis, Indiana, USA; ${ }^{2}$ Indiana University, Indiana, USA

Background Age-specific incidence rates of sexually transmitted infections (STI) with Chlamydia trachomatis (CT), Neisseria gonorrhoeae (GC), and Trichomonas vaginalis (TV) are not well characterised in adolescents and young adult women. In this research, we described the CT, GC, and TV incidence rates in young women as functions of age.

Methods Young women aged 14 and 17 were recruited from three adolescent medicine clinics in Indianapolis, Indiana. Study participants were followed longitudinally for up to 8.2 years. Participants were tested for CT, GC, and TV at the time of enrolment, and at subsequent quarterly visits. Infected individuals were treated at or shortly after each visit. We analysed the longitudinally measured incidence infections with CT, GC, and TV using generalised additive mixed effect models (GAMM) with a logit link for binary outcomes. The age effect on STI incidence was modelled as a smooth nonlinear function in the GAMM analysis and a random subject effect was included to accommodate the correlations among repeated STI assessments within each individual. Estimated incidence rates (with $95 \%$ CIs, in colour) for each organism were reported as smooth functions of age.

Results The cohort included 386 young women. The average age at enrolment of study participants was 15.3 years ( $S D=1.1$ years). A majority (89.1\%) of study participants were African-American. The average age at first sexual intercourse was 14.2 years $(\mathrm{SD}=2$, median $=14$ years). The mean cumulative number of sexual partners measured at the time enrolment was $3(\mathrm{SD}=4$, median=2). The average length of follow-up was 3.5 years ( $\mathrm{SD}=2.2$ years). Baseline prevalence rates were $10.9 \%, 4.4 \%$ and $6.0 \%$ for CT, GC, and TV, respectively. The age-specific incidence rates for the three organisms and any STI are seen in the Abstract P1-S1.12 figure 1.
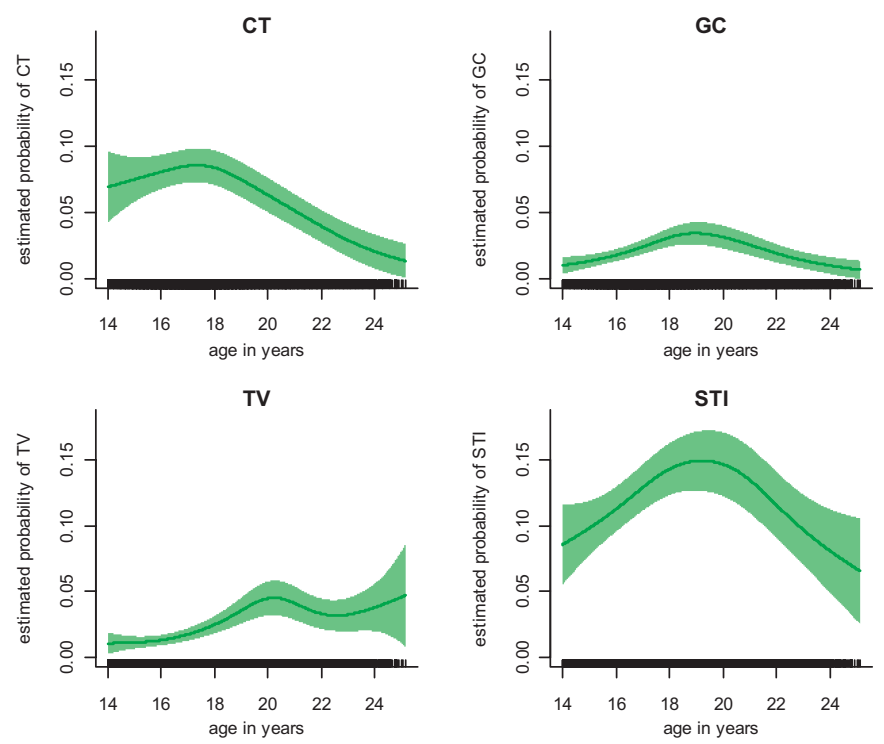

Abstract P1-S1.12 Figure 1 WTU.

Conclusions The estimated STI incidence rates clearly differ by organisms, not only in magnitude but also in peak age. While the prevalence rates of the respective organisms in the partner population may be a contributor to the differential risk of STI acquisition, the fact that such differences were observed from the same group of individuals with the same sexual partners and sexual behaviours raises questions about age-related differences in susceptibility to infection by the three organisms.

\section{Epidemiology poster session 1: STI trends-HIV}

\section{P1-S1.13 HETEROGENEITY OF THE HIV EPIDEMIC IN THE GENERAL POPULATION OF KARNATAKA STATE, INDIA}

doi:10.1136/sextrans-2011-050108.13

${ }^{1} \mathrm{P}$ Banandur, ${ }^{2} \mathrm{R}$ S Potty, ${ }^{3} \mathrm{~S}$ B Mahagaonkar, ${ }^{4} \mathrm{~J}$ Bradley, ${ }^{5} \mathrm{R}$ M Banadakoppa, ${ }^{6} \mathrm{R}$ G Washington, ${ }^{7} \mathrm{~J}$ F Blanchard, ${ }^{7} \mathrm{~S}$ Moses, ${ }^{8} \mathrm{C} \mathrm{M}$ Lowndes, ${ }^{9} \mathrm{M}$ Alary. ${ }^{7} \mathrm{CHARME}$ ॥ Project, Rajarajeswari Medical College and Hospital, Bangalore, India; ${ }^{2}$ CHARME II Project, Bangalore, India; ${ }^{3} \mathrm{CHARME}$ I Project, India, Karuna Medical College, India; ${ }^{4}$ CHARME I Project, Bangalore, India; ${ }^{5}$ Karnataka Health Promotion Trust, Bangalore, India; ${ }^{6}$ St Johns Research Institute, India; ${ }^{7}$ University of Manitoba, Winnipeg, Canada; ${ }^{8}$ Health Protection Agency, UK; ${ }^{9}$ Centre hospitalier affilié universitaire de Québec, Quebec, Canada

Background In the context of AVAHAN-The India AIDS Initiative of the Bill and Melinda Gates Foundation, general population surveys (GPS) have been carried out in three Karnataka districts in India. Given the north-south gradient already observed in HIV 